\section{Leistungssport: Mit Vorsorge-Screenings den plötzlichen Herztod verhindern}

\begin{abstract}
Ein akuter Herztod bei Leistungssportlern findet immer eine große mediale Resonanz. Dann wird die Frage gestellt: Hätte dieses fatale Ereignis durch ein Vorsorge-Screening verhindert werden können?
\end{abstract}

$\mathrm{D}$ as Paradoxe beim Sport ist, dass er eigentlich gesundheitsfördernd ist, im Einzelfall aber zum akuten Herztod führen kann", sagte Prof. Andreas Schuchert, Neumünster. Doch mit 4,6 Ereignissen pro 1 Millionen Einwohner pro Jahr handele es sich um ein sehr seltenes Ereignis; zu ca. 95\% sind Männer betroffen. Doch bei Wettkampfsportlern sei das Risiko erhöht. Wenn man über ein Vorsorge-Screening spreche, müsse man zwischen jungen Wettkampfsportlern unter 35 Jahren und über 35-jährigen Freizeitsportlern unterscheiden.

Daher muss man als Ursache bei jungen Wettkampfsportlern vorrangig an eine Myokarditis, hypertrophe Kardiomyopathie (HCM) und Ionenkanalerkrankung denken, bei älteren Sportlern primär an eine KHK oder Aortenstenose. „Doch sowohl bei jungen als auch bei älteren Patienten bleibt in circa 30 Prozent der Fälle die Ursache unklar", so Schuchert.

\section{Altersabhängige Strategie!}

Beim jungen Wettkampfsportler geht es vorrangig darum, eine genetische Erkrankung wie HCM, arrhythmogene rechtsventrikuläre Kardiomyopathie (ARVC) oder Ionenkanalerkrankung früh zu erfassen. Dies gelingt häufig mit dem Ruhe-EKG. Zeigt dieses relevante Auffälligkeiten oder bei einer Familienanamnese für den akuten Herztod, sollte eine Farbdopplerechokardiografie und evtl. eine Ergometrie erfolgen.

Zur Detektion einer Koronaranomalie bietet sich heute das Cardio-MRT an.
„Ab dem 35. Lebensjahr sollte auch bei Freizeitsportlern immer ein BelastungsEKG durchgeführt werden“, empfiehlt Schuchert.

\section{EKG-Diagnostik bei Kanalopathie}

Die häufigsten Ionenkanalerkrankungen sind das Long-QT-Syndrom (LQTS), das Short-QT-Syndrom (SQTS), das Brugada-Syndrom und die polymorphe katecholaminerge Kammertachykardie. Die Diagnose ergibt sich meist aus der Eigen- und Familienanamnese, dem EKG-Befund und womöglich einer molekulargenetischen Untersuchung. „Das Erkennen einer Kanalopathie aus dem Oberflächen-EKG ist nicht immer einfach", sagte Dr. Rainer Schimpf, Ludwigshafen. Schon die genaue Bestimmung der QT-Zeit, genauer gesagt die Abgrenzung der T-Welle zur Erfassung eines Long-QT-Syndroms könne Probleme bereiten. Es empfiehlt sich, an den absteigenden Schenkel der T-Welle eine Tangente zu legen. Ein LQTS ist bei einer QT-Zeit $\geq 480 \mathrm{msec}$ zu diskutieren.

In unklaren Fällen sollte ein Stand upTest erfolgen. Dabei wird nach fünfminütigem Liegen ein EKG abgeleitet, um die minimale Herzfrequenz zu erfassen. Nach dem Aufstehen steigen Herzfrequenz und QT-Zeit an, bei einem LQTS aber stärker und länger als bei Gesunden. Dazu kommen Veränderungen der T-Welle. „Dieser einfache Test hat eine Sensitivität von fast 95 Prozent bei einer Spezifität von 79 Prozent", sagte Schimpf.

Das entscheidende Kriterium für das SQTS ist eine QT-Zeit $\leq 340 \mathrm{msec}$.
Typisch für das Brugada-Syndrom ist die konvexbogige ST-Hebung in V1 und V2. Ist diese jedoch sattelförmig, ist diese Hebung nicht als Brugada-Syndrom zu bewerten. In unklaren Fällen sollten zusätzlich rechts- und linksparasternale EKG-Ableitungen erfolgen. Auch mittels Ajmalin-Test lässt sich das Brugada-Syndrom demaskieren.

\section{ICD bei symptomatischen Patienten}

Laut Prof. Wilhelm Haverkamp aus Berlin ist „der ICD bei symptomatischen Patienten mit einer Kanalopathie heute die Therapie der Wahl“. Nur bei asymptomatischen Patienten sei nach sorgfältiger Risikostratifizierung eine medikamentöse Therapie sinnvoll, evtl. in Kombination mit einem ICD. Selbst für Patienten, die einen ICD ablehnten, sei die medikamentöse Therapie unverzichtbar.

Das Medikament der ersten Wahl beim LQTS sind Betablocker; am besten spricht der Typ 1 darauf an. „Auch wirken nicht alle Betablocker gleich gut", so Haverkamp. Am effektivsten sind Propranolol und Nadolol. Metoprolol sei sehr viel schlechter wirksam. Dabei sollte man möglichst hohe Dosen einsetzen. Eine Bradykardie ist keine Kontraindikation. Im Einzelfall können abhängig vom LQTS-Typ auch andere Antiarrhythmika zum Einsatz kommen. Das Mittel der Wahl beim Brugada-Syndrom ist Chinidin, eine Alternative ist Isoproterenol. Unverzichtbar ist Chinidin als Notfallmedikament beim elektrischen Sturm, also rezidivierenden ventrikulären Tachykardien (VT), und bei ICD-Patienten mit häufigen $\mathrm{VT}$, rezidivierendem Vorhofflimmern oder supraventrikulären Tachykardien.

Andere Klasse I-Antiarrhythmika sind ebenso wie Antipsychotika und Anästhetika wie Propofol kontraindiziert. Bei der katecholaminergen polymorphen Kammertachykardie sind Betablocker und Flecainid wirksam. 